Original Research Article

\title{
Antibiotic resistance pattern in intensive care unit of a tertiary care teaching hospital
}

\author{
Amit Bhatia $^{1}$, Juhi Kalra ${ }^{1 *}$, Saurabh Kohli ${ }^{1}$, Barnali Kakati ${ }^{2}$, Reshma Kaushik $^{3}$
}

${ }^{1}$ Department of Pharmacology, ${ }^{2}$ Department of Microbiology, ${ }^{3}$ Department of General Medicine, Himalayan Institute of Medical Sciences, Swami Rama Himalayan University, Dehradun, Uttarakhand, India

Received: 16 February 2018 Accepted: 28 March 2018

\section{*Correspondence to:}

Dr. Juhi Kalra,

Email: drjuhi9@gmail.com

Copyright: (C) the author(s), publisher and licensee Medip Academy. This is an openaccess article distributed under the terms of the Creative Commons Attribution NonCommercial License, which permits unrestricted noncommercial use, distribution, and reproduction in any medium, provided the original work is properly cited.

\begin{abstract}
Background: Antimicrobials are a major class of drugs prescribed in Intensive Care Unit (ICU). Widespread use of empirical antibiotic therapy has facilitated the emergence of drug resistance, since empirical therapy is very often initiated at the outset, even before culture and sensitivity reports are available. The problem of drug resistance is on a rise, therefore, this study was planned to assess the drug resistance and sensitivity patterns of the blood isolates recovered from ICU.

Methods: An observational- prospective study was conducted in the Tertiary care teaching hospital over a period of twelve months to assess antibiotic resistance and sensitivity pattern. A total of 104 consecutive patients receiving antibiotics in the ICU and having blood cultures with significant growth were included in the study. Blood sample was collected and after obtaining a culture growth, the identification and antimicrobial sensitivity testing was done.

Results: Blood stream infection by Gram-negative bacteria $(50.96 \%)$ was more common than Gram-positive bacteria (49.04\%). Coagulase negative Staphylococci (CoNS) was the predominant single blood culture isolate (35.58\%). Klebsiella pneumoniae (13.46\%), Escherichia coli (12.50\%), Acinetobacter baumannii complex $(7.69 \%)$ were commonly isolated gram negative organisms. Gram positive isolates were resistant to beta lactams in maximum patients whereas Tigecycline, Linezolid, Daptomycin, Vancomycin, Nitrofurantoin and Teicoplanin were sensitive against them. Common gram negative isolates were sensitive to Colistin and Tigecycline but resistant to most of the antibiotics.

Conclusions: A preponderance of gram negative bacteria over gram positive bacteria was noted with a higher degree of resistance to most of the first line antimicrobial agents.
\end{abstract}

Keywords: Antibiotics, Blood stream infection, Intensive care unit, Resistance and sensitivity pattern

\section{INTRODUCTION}

Antimicrobials is a general term for natural or synthetic compounds which at certain concentrations inhibit growth of or kill microorganisms. ${ }^{1}$ Antibiotics are the most frequently prescribed drugs in Intensive Care Unit. ${ }^{2}$ One of the important aspects of effective treatment of lifethreatening infections leading to sepsis and septic shock is prescription of appropriate antimicrobial therapy. ${ }^{3}$
The frequent use and misuse of certain agents (or classes of antimicrobial agents) in a hospital or other health care setting can result in selection of organisms that are resistant to that particular antibiotic. ${ }^{4}$ Around $20-50 \%$ of all antibiotics use was estimated to be inappropriate. ${ }^{5}$ Resistance of microorganism to an antimicrobial agents that were originally effective for treatment of infections caused by it, is called as antimicrobial resistance. ${ }^{6}$ The resistance in pathogenic bacteria to several antimicrobial 
agents i.e. multidrug resistance has lead to a major public health problem as there are currently very few effective antibiotic agents for infections caused by the bacteria. Multidrug-Resistance is defined as acquired resistance to at least one antibiotic among three or more antibiotic categories. $^{7}$

For finding out the susceptibility or resistance, Culture and sensitivity and the Minimum Inhibitory Concentration (MIC) testing is done. The Minimum Inhibitory Concentration (MIC) is defined as the minimum concentration of an antibiotic agent which completely inhibits the growth of an organism. Clinical microbiology laboratories have categorized microorganisms as clinically susceptible (S), intermediate (I) or resistant (R) on the basis of clinical breakpoints determined in a well-defined standard test system. Clinical Breakpoints are discriminatory antimicrobial concentrations, which are used in the interpretation of susceptibility testing results. ${ }^{8}$

Antimicrobial resistance is a major public health problem in India. It is known that the infectious disease burden in India is among the highest in the world. It is also known that susceptibility patterns vary from region to region. Since there is wide diversity in the prevalence of predominant pathogens and their antimicrobial susceptibilities, especially within individual ICUs, hence local susceptibility patterns help in rational prescribing of antimicrobials and can delay the emergence of antimicrobial resistance.

An ICU setting is a major source of emergence of the drug resistance as empirical antibiotic therapy is very often initiated at the outset, even before culture and sensitivity reports are available. ${ }^{9}$ Therefore, choice and monitoring of antibiotic therapy is of utmost importance to prevent emergence of antibiotic resistance. ${ }^{10}$ Authors therefore planned this study to assess the drug resistance and sensitivity patterns in ICU of a tertiary care teaching hospital.

Aims and objectives of the study was to study the drug resistance and sensitivity patterns of the blood isolates recovered from ICU.

\section{METHODS}

The study was conducted in the Tertiary care teaching hospital after obtaining the written informed consent from the patients and the ethical approval from the Ethics Committee over a period of twelve months.

\section{Study design}

It was an Observational prospective study. One hundred and four consecutive patients receiving antibiotics in the ICU and having blood cultures with significant growth were included in the study.

\section{Inclusion criteria}

- $\quad$ All the consecutive patients from the Intensive Care Unit that were prescribed antibiotics and whose blood cultures yield bacterial isolates.

\section{Exclusion criteria}

- $\quad$ Patients admitted in paediatric and neonatal ICUs.

- Patients from the Intensive Care Unit that were prescribed antibiotics and whose blood cultures yield fungal isolates.

The present study was an observational prospective study done to assess the drug resistance and sensitivity patterns of the blood isolates recovered from ICU. One hundred and four consecutive patients receiving antibiotics in the ICU and having blood cultures with significant growth were included in the study after taking the written informed consent. Hence, the blood culture and sensitivity reports of 104 patients were analysed. After enrolment, their details regarding demography, bacteria isolated, culture and sensitivity reports and the details of prescribed antibiotics were recorded in the case recording form. Laboratory investigations were carried out by BACTEC 9120 and VITEK 2. Blood sample was collected maintaining all the aseptic precautions and was inoculated in aerobic BACTEC bottle for culture. The inoculated bottle was then incubated in BACTEC 9120 for obtaining growth. ${ }^{11}$ On obtaining a culture growth, the identification and antimicrobial sensitivity testing was done by VITEK $2 .^{12}$

The primary end point was to study the drug resistance and sensitivity patterns of blood isolates from patients prescribed with antibiotics in the ICU and having blood cultures with significant growth (based on Culture/Sensitivity reports).

\section{Data management and statistical analysis}

Interpretation and analysis of the results were carried out using software Microsoft Excel 2007 and SPSS version 20.0. Demographic profile of patients, isolation pattern of various organisms and their antibiograms and prescription pattern of antibiotics were analyzed by using descriptive statistics. Descriptive analysis was represented by graphical representation wherever required using Microsoft excel 2007.

\section{RESULTS}

Among the 104 patients included, $58(55.77 \%)$ were male patients and $46(44.23 \%)$ female patients. The mean age of the patients was $51.95 \pm 16.83$ years (Figure 1 ).

Among the isolates recovered from the blood cultures of 104 patients, 51 isolates $(49.04 \%)$ were Gram positive bacteria while $53(50.96 \%)$ were Gram negative bacteria. Among gram positive isolates, Coagulase negative 
staphylococci (CoNS) was the most common blood culture isolate $(35.58 \%)$. The CoNS include, Staphylococcus haemolyticus, Staphylococcus epidermidis, Staphylococcus hominis, Staphylococcus warneri, Staphylococcus capitis, Staphylococcus lentus. Among the gram negative isolates, most commonly isolated organisms were Klebsiella pneumoniae (13.46\%), Escherichia coli (12.50\%), Acinetobacter baumannii complex (7.69\%) (Figure 2).

Among the Gram-positive isolates (GPIs), Staphylococcus hemolyticus was resistant to Benzylpenicillin, Ciprofloxacin, Oxacillin and Erythromycin and sensitive to Tigecycline, Linezolid, Daptomycin, Vancomycin and Nitrofurantoin in all patients. Staphylococcus epidermidis was resistant to Benzylpenicillin and sensitive to Linezolid, Daptomycin, Teicoplanin, Vancomycin and Nitrofurantoin in all patients. In case of Staphylococcus hominis, resistance against Benzylpenicillin, Ciprofloxacin, Oxacillin, Erythromycin and Levofloxacin was seen in $87 \%$ patients while it was sensitive to Tigecycline, Linezolid, Daptomycin and Nitrofurantoin in all patients. Staphylococcus aureus was resistant to Benzylpenicillin and Ciprofloxacin and sensitive to Tigecycline, Linezolid, Daptomycin, Teicoplanin and Vancomycin in all patients. S. aureus and CoNS were found to be resistant to methicillin in $67 \%$ and $94 \%$ patients respectively (Table 1).

Among the Gram-negative isolates (GNIs), Klebsiella pneumoniae isolates were resistant to Beta - lactams, Cephalosporins in all patients. It showed sensitivity against Colistin and Tigecycline in $78 \%$ and $80 \%$ patients respectively. Escherichia coli was resistant to Cefadroxil, Piperacillin - Sulbactam and Nalidixic acid and sensitive to Minocycline, Ertapenem, Amikacin, Colistin and
Tigecycline in all patients. Acinetobacter baumannii complex was resistant to Cefadroxil, Piperacillin Sulbactam, Nalidixic acid, Aztreonam, Cefuroxime and Ceftriaxone in all patients. It was found to be sensitive against Colistin and Tigecycline in all and $87 \%$ patients respectively (Table 2).

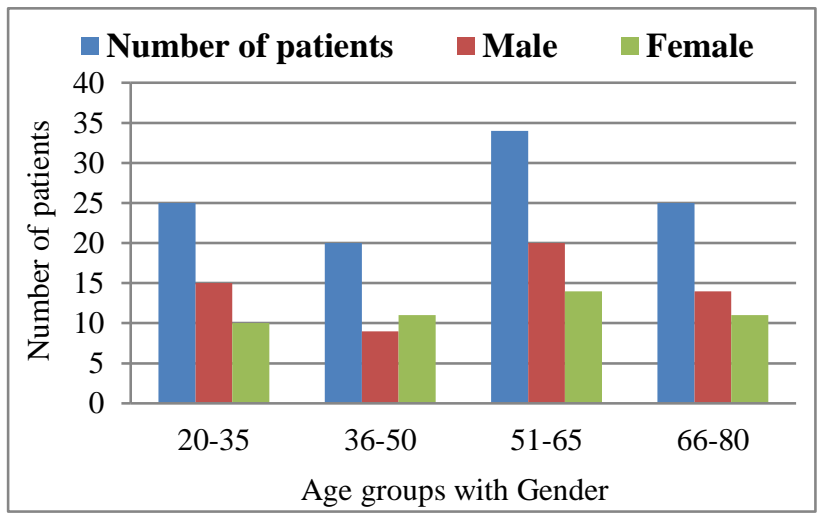

Figure 1: Demographic characteristics of the patients $(n=104)$.

The most frequently used antibiotic drugs in 104 patients admitted in ICU were Piperacillin - Tazobactam (50\%) followed by Meropenem (34.62\%), Metronidazole (34.62\%), Linezolid (22.12\%), Azithromycin (20.19\%), Colistin (16.35\%), Doxycycline (16.35\%), Amoxycillin Clavulanic acid (14.42\%), Levofloxacin (13.46\%), Polymyxin B (13.46\%) and Tigecycline $(13.46 \%)$. However, many other antibiotics were also prescribed from different classes such as Anti-tubercular drugs, Aminoglycosides, Glycopeptides, Lincosamide, Diaminopyrimidines - Sulphonamides, Nitrobenzene (Table 3).

Table 1: Antibiogram of common Gram-positive isolates (GPIs) as per the blood Culture/Sensitivity reports of ICU patients.

\begin{tabular}{|c|c|c|c|c|c|c|c|c|c|c|c|c|c|c|c|c|c|c|c|c|c|c|c|c|}
\hline Isolates & Stapl & lococ & s ha & nolytic & & & Stap & loco & us e & derm & & & & hyloc & cus & omin & & & Stap & yloce & us a & eus & & \\
\hline Susceptibility & $\begin{array}{l}\mathbf{R} \\
\%\end{array}$ & $\begin{array}{l}\mathbf{R} \\
(\mathbf{n})\end{array}$ & $\begin{array}{l}\text { I } \\
\%\end{array}$ & $\begin{array}{l}\text { I } \\
\text { (n) }\end{array}$ & $\begin{array}{l}\text { S } \\
\%\end{array}$ & $\begin{array}{l}\text { S } \\
\text { (n) }\end{array}$ & $\begin{array}{l}\mathbf{R} \\
\%\end{array}$ & $\begin{array}{l}\mathbf{R} \\
\text { (n) }\end{array}$ & $\begin{array}{l}\text { I } \\
\%\end{array}$ & $\begin{array}{l}\text { I } \\
\text { (n) }\end{array}$ & $\begin{array}{l}\mathrm{S} \\
\%\end{array}$ & $\begin{array}{l}\text { S } \\
\text { (n) }\end{array}$ & $\begin{array}{l}\mathbf{R} \\
\%\end{array}$ & $\begin{array}{l}\text { R } \\
\text { (n) }\end{array}$ & $\begin{array}{l}\text { I } \\
\%\end{array}$ & $\begin{array}{l}\text { I } \\
\text { (n) }\end{array}$ & $\begin{array}{l}\text { S } \\
\%\end{array}$ & $\begin{array}{l}\mathbf{S} \\
\text { (n) }\end{array}$ & $\begin{array}{l}\mathbf{R} \\
\%\end{array}$ & $\begin{array}{l}\mathbf{R} \\
\text { (n) }\end{array}$ & $\begin{array}{l}\text { I } \\
\%\end{array}$ & $\begin{array}{l}\text { I } \\
\text { (n) }\end{array}$ & $\begin{array}{l}\mathrm{S} \\
\%\end{array}$ & $\begin{array}{l}\text { S } \\
\text { (n) }\end{array}$ \\
\hline Benzylpenicillin & 100 & $\begin{array}{l}15 \\
(15)\end{array}$ & & & & & 100 & $\begin{array}{l}9 \\
\text { (9) }\end{array}$ & & & & & 87 & $\begin{array}{l}7 \\
(8)\end{array}$ & & & 13 & $\begin{array}{l}1 \\
\text { (8) }\end{array}$ & 100 & $\begin{array}{l}6 \\
\text { (6) }\end{array}$ & & & & \\
\hline Ciprofloxacin & 100 & $\begin{array}{l}15 \\
(15)\end{array}$ & & & & & 67 & $\begin{array}{l}6 \\
(9)\end{array}$ & 22 & $\begin{array}{l}2 \\
(9)\end{array}$ & 11 & $\begin{array}{l}1 \\
\text { (9) }\end{array}$ & 87 & $\begin{array}{l}7 \\
(8)\end{array}$ & & & 13 & $\begin{array}{l}1 \\
(8)\end{array}$ & 100 & $\begin{array}{l}4 \\
(4)\end{array}$ & & & & \\
\hline Cefoxitin & 100 & $\begin{array}{l}15 \\
(15)\end{array}$ & & & & & 89 & $\begin{array}{l}8 \\
(9)\end{array}$ & & & 11 & $\begin{array}{l}1 \\
(9)\end{array}$ & 87 & $\begin{array}{l}7 \\
(8)\end{array}$ & & & 13 & $\begin{array}{l}1 \\
(8)\end{array}$ & 67 & $\begin{array}{l}4 \\
(6)\end{array}$ & & & 33 & $\begin{array}{l}2 \\
(6)\end{array}$ \\
\hline Oxacillin & 100 & $\begin{array}{l}15 \\
\text { (15) }\end{array}$ & & & & & 89 & $\begin{array}{l}8 \\
(9)\end{array}$ & & & 11 & $\begin{array}{l}1 \\
\text { (9) }\end{array}$ & 87 & $\begin{array}{l}7 \\
(8)\end{array}$ & & & 13 & $\begin{array}{l}1 \\
(8)\end{array}$ & 80 & $\begin{array}{l}4 \\
(5)\end{array}$ & & & 20 & $\begin{array}{l}1 \\
(5)\end{array}$ \\
\hline Erythromycin & 100 & $\begin{array}{l}15 \\
(15)\end{array}$ & & & & & 78 & $\begin{array}{l}7 \\
(9)\end{array}$ & & & 22 & $\begin{array}{l}2 \\
(9)\end{array}$ & 87 & $\begin{array}{l}7 \\
(8)\end{array}$ & & & 13 & $\begin{array}{l}1 \\
(8)\end{array}$ & 50 & $\begin{array}{l}2 \\
\text { (4) }\end{array}$ & & & 50 & $\begin{array}{l}2 \\
(4)\end{array}$ \\
\hline Levofloxacin & 67 & $\begin{array}{l}10 \\
(15)\end{array}$ & 27 & $\begin{array}{l}4 \\
(15)\end{array}$ & 6 & $\begin{array}{l}1 \\
(15)\end{array}$ & 45 & $\begin{array}{l}4 \\
(9)\end{array}$ & 44 & $\begin{array}{l}4 \\
(9)\end{array}$ & 11 & $\begin{array}{l}1 \\
(9)\end{array}$ & 87 & $\begin{array}{l}7 \\
(8)\end{array}$ & & & 13 & $\begin{array}{l}1 \\
(8)\end{array}$ & 75 & $\begin{array}{l}3 \\
(4)\end{array}$ & 25 & $\begin{array}{l}1 \\
(4)\end{array}$ & & \\
\hline Tigecycline & & & & & 100 & $\begin{array}{l}11 \\
\text { (11) }\end{array}$ & 11 & $\begin{array}{l}1 \\
\text { (9) }\end{array}$ & & & 89 & $\begin{array}{l}8 \\
\text { (9) }\end{array}$ & & & & & 100 & $\begin{array}{l}8 \\
(8)\end{array}$ & & & & & 100 & $\begin{array}{l}5 \\
(5)\end{array}$ \\
\hline Linezolid & & & & & 100 & $\begin{array}{l}12 \\
(12)\end{array}$ & & & & & 100 & $\begin{array}{l}7 \\
\text { (7) } \\
\end{array}$ & & & & & 100 & $\begin{array}{l}6 \\
(6) \\
\end{array}$ & & & & & 100 & $\begin{array}{l}4 \\
\text { (4) }\end{array}$ \\
\hline Daptomycin & & & & & 100 & $\begin{array}{l}15 \\
(15)\end{array}$ & & & & & 100 & $\begin{array}{l}7 \\
\text { (7) }\end{array}$ & & & & & 100 & $\begin{array}{l}7 \\
\text { (7) }\end{array}$ & & & & & 100 & $\begin{array}{l}4 \\
\text { (4) }\end{array}$ \\
\hline Teicoplanin & & & 6 & $\begin{array}{l}1 \\
(15)\end{array}$ & 94 & $\begin{array}{l}14 \\
(15)\end{array}$ & & & & & 100 & $\begin{array}{l}9 \\
\text { (9) }\end{array}$ & 13 & $\begin{array}{l}1 \\
(8)\end{array}$ & & & 87 & $\begin{array}{l}7 \\
(8)\end{array}$ & & & & & 100 & $\begin{array}{l}5 \\
(5) \\
\end{array}$ \\
\hline Vancomycin & & & & & 100 & $\begin{array}{l}15 \\
(15)\end{array}$ & & & & & 100 & $\begin{array}{l}9 \\
(9)\end{array}$ & 13 & $\begin{array}{l}1 \\
(8)\end{array}$ & & & 87 & $\begin{array}{l}7 \\
(8)\end{array}$ & & & & & 100 & $\begin{array}{l}5 \\
(5)\end{array}$ \\
\hline Nitrofurantoin & & & & & 100 & $\begin{array}{l}9 \\
(9)\end{array}$ & & & & & 100 & $\begin{array}{l}5 \\
\text { (5) }\end{array}$ & & & & & 100 & $\begin{array}{l}4 \\
\text { (4) }\end{array}$ & & & 50 & $\begin{array}{l}1 \\
(2)\end{array}$ & 50 & $\begin{array}{l}1 \\
\text { (2) }\end{array}$ \\
\hline
\end{tabular}


Table 2: Antibiogram of common gram-negative isolates (GNIs) as per the blood Culture/Sensitivity reports of ICU patients.

\begin{tabular}{|c|c|c|c|c|c|c|c|c|c|c|c|c|c|c|c|c|c|c|}
\hline Isolates & Kleb & ella p & eum & niae & & & Esch & richia & coli & & & & Acin & toba & ter $\mathbf{b}$ & uma & nii co & nplex \\
\hline Susceptibility & $\begin{array}{l}\mathbf{R} \\
\%\end{array}$ & $\begin{array}{l}\mathbf{R} \\
(\mathbf{n})\end{array}$ & $\begin{array}{l}\text { I } \\
\%\end{array}$ & $\begin{array}{l}\text { I } \\
\text { (n) }\end{array}$ & $\begin{array}{l}S \\
\%\end{array}$ & $\begin{array}{l}\mathbf{S} \\
(\mathbf{n})\end{array}$ & $\begin{array}{l}\mathbf{R} \\
\%\end{array}$ & $\begin{array}{l}\mathbf{R} \\
(\mathbf{n})\end{array}$ & $\begin{array}{l}\text { I } \\
\%\end{array}$ & $\begin{array}{l}\text { I } \\
\text { (n) }\end{array}$ & $\begin{array}{l}S \\
\%\end{array}$ & $\begin{array}{l}\text { S } \\
\text { (n) }\end{array}$ & $\begin{array}{l}\mathbf{R} \\
\%\end{array}$ & $\begin{array}{l}\mathbf{R} \\
(\mathbf{n})\end{array}$ & $\begin{array}{l}\text { I } \\
\%\end{array}$ & $\begin{array}{l}\text { I } \\
(\mathbf{n})\end{array}$ & $\begin{array}{l}\mathrm{S} \\
\%\end{array}$ & $\mathbf{S}(\mathbf{n})$ \\
\hline Cefadroxil & 100 & $\begin{array}{l}3 \\
(3)\end{array}$ & & & & & 100 & $\begin{array}{l}8 \\
(8)\end{array}$ & & & & & 100 & $\begin{array}{l}1 \\
(1)\end{array}$ & & & & \\
\hline $\begin{array}{l}\text { Piperacillin - } \\
\text { sulbactam }\end{array}$ & 100 & $\begin{array}{l}14 \\
(14)\end{array}$ & & & & & 100 & $\begin{array}{l}1 \\
(1)\end{array}$ & & & & & 100 & $\begin{array}{l}1 \\
(1)\end{array}$ & & & & \\
\hline Nalidixic acid & 100 & $\begin{array}{l}12 \\
(12)\end{array}$ & & & & & 100 & $\begin{array}{l}9 \\
(9)\end{array}$ & & & & & 100 & $\begin{array}{l}2 \\
(2)\end{array}$ & & & & \\
\hline Aztreonam & 100 & $\begin{array}{l}3 \\
(3)\end{array}$ & & & & & 91 & $\begin{array}{l}11 \\
(12)\end{array}$ & & & 9 & $\begin{array}{l}1 \\
(12)\end{array}$ & 100 & $\begin{array}{l}7 \\
(7)\end{array}$ & & & & \\
\hline Cefuroxime & 100 & $\begin{array}{l}13 \\
(13)\end{array}$ & & & & & 89 & $\begin{array}{l}8 \\
(9)\end{array}$ & & & 11 & $\begin{array}{l}1 \\
(9)\end{array}$ & 100 & $\begin{array}{l}2 \\
(2)\end{array}$ & & & & \\
\hline Ceftriaxone & 100 & $\begin{array}{l}13 \\
(13)\end{array}$ & & & & & 89 & $\begin{array}{l}8 \\
(9)\end{array}$ & & & 11 & $\begin{array}{l}1 \\
(9)\end{array}$ & 100 & $\begin{array}{l}2 \\
(2)\end{array}$ & & & & \\
\hline Cefepime & 100 & $\begin{array}{l}14 \\
(14)\end{array}$ & & & & & 92 & $\begin{array}{l}12 \\
(13)\end{array}$ & & & 8 & $\begin{array}{l}1 \\
(13)\end{array}$ & 87 & $\begin{array}{l}7 \\
(8)\end{array}$ & & & 13 & $1(8)$ \\
\hline Ciprofloxacin & 100 & $\begin{array}{l}14 \\
(14)\end{array}$ & & & & & 85 & $\begin{array}{l}11 \\
(13)\end{array}$ & & & 15 & $\begin{array}{l}2 \\
(13)\end{array}$ & 87 & $\begin{array}{l}7 \\
(8)\end{array}$ & & & 13 & $1(8)$ \\
\hline Gentamicin & 93 & $\begin{array}{l}13 \\
(14)\end{array}$ & & & 7 & $\begin{array}{l}1 \\
(14)\end{array}$ & 62 & $\begin{array}{l}8 \\
(13)\end{array}$ & & & 38 & $\begin{array}{l}5 \\
(13)\end{array}$ & 87 & $\begin{array}{l}7 \\
(8)\end{array}$ & & & 13 & $1(8)$ \\
\hline $\begin{array}{l}\text { Piperacillin - } \\
\text { Tazobactam }\end{array}$ & 100 & $\begin{array}{l}14 \\
(14)\end{array}$ & & & & & 23 & $\begin{array}{l}3 \\
(13)\end{array}$ & 8 & $\begin{array}{l}1 \\
(13)\end{array}$ & 69 & $\begin{array}{l}9 \\
(13)\end{array}$ & 87 & $\begin{array}{l}7 \\
(8)\end{array}$ & & & 13 & $1(8)$ \\
\hline Imipenem & 100 & $\begin{array}{l}14 \\
(14)\end{array}$ & & & & & 8 & $\begin{array}{l}1 \\
(13)\end{array}$ & & & 92 & $\begin{array}{l}12 \\
(13)\end{array}$ & 74 & $\begin{array}{l}6 \\
(8)\end{array}$ & 13 & $\begin{array}{l}1 \\
(8)\end{array}$ & 13 & $1(8)$ \\
\hline Meropenem & 100 & $\begin{array}{l}14 \\
(14)\end{array}$ & & & & & 8 & $\begin{array}{l}1 \\
(13)\end{array}$ & & & 92 & $\begin{array}{l}12 \\
(13)\end{array}$ & 74 & $\begin{array}{l}6 \\
(8)\end{array}$ & 13 & $\begin{array}{l}1 \\
(8)\end{array}$ & 13 & $1(8)$ \\
\hline Levofloxacin & 100 & $\begin{array}{l}8 \\
(8)\end{array}$ & & & & & 62 & $\begin{array}{l}5 \\
(8)\end{array}$ & & & 38 & $\begin{array}{l}3 \\
(8)\end{array}$ & 68 & $\begin{array}{l}4 \\
(6)\end{array}$ & 16 & $\begin{array}{l}1 \\
(6)\end{array}$ & 16 & $1(6)$ \\
\hline Minocycline & 100 & $\begin{array}{l}1 \\
(1)\end{array}$ & & & & & & & & & 100 & $\begin{array}{l}4 \\
(4)\end{array}$ & 17 & $\begin{array}{l}1 \\
(6)\end{array}$ & 33 & $\begin{array}{l}2 \\
(6)\end{array}$ & 50 & $3(6)$ \\
\hline Ertapenem & 100 & $\begin{array}{l}6 \\
(6)\end{array}$ & & & & & & & & & 100 & $\begin{array}{l}9 \\
(9)\end{array}$ & & & & & & \\
\hline Amikacin & 93 & $\begin{array}{l}13 \\
(14)\end{array}$ & 7 & $\begin{array}{l}1 \\
(14)\end{array}$ & & & & & & & 100 & $\begin{array}{l}13 \\
\text { (13) }\end{array}$ & & & & & & \\
\hline Colistin & 22 & $\begin{array}{l}3 \\
\text { (14) }\end{array}$ & & & 78 & $\begin{array}{l}11 \\
(14)\end{array}$ & & & & & 100 & $\begin{array}{l}13 \\
\text { (13) }\end{array}$ & & & & & 100 & $8(8)$ \\
\hline Tigecycline & 10 & $\begin{array}{l}1 \\
(10)\end{array}$ & 10 & $\begin{array}{l}1 \\
(10)\end{array}$ & 80 & $\begin{array}{l}8 \\
(10)\end{array}$ & & & & & 100 & $\begin{array}{l}13 \\
(13)\end{array}$ & & & 13 & $\begin{array}{l}1 \\
(8)\end{array}$ & 87 & $7(8)$ \\
\hline
\end{tabular}

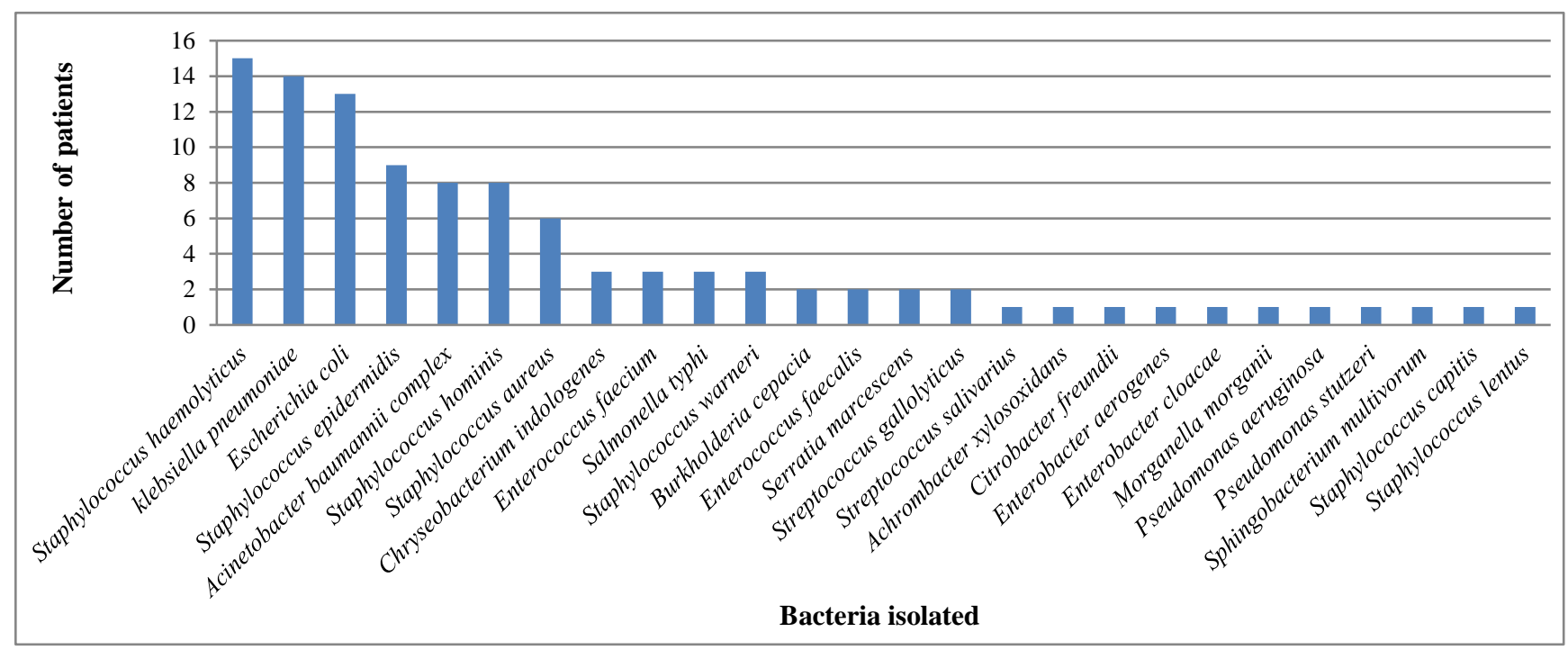

Figure 2: Bacteria isolated from blood cultures obtained from ICU patients $(n=104)$. 
Table 3: Commonly prescribed antibiotic drugs in Intensive Care Unit ( $\mathbf{n}=104)$.

\begin{tabular}{|ll|}
\hline $\begin{array}{l}\text { Commonly prescribed } \\
\text { antibiotics }\end{array}$ & $\begin{array}{l}\text { Number of patients } \\
\text { prescribed the antibiotic } \\
{[\mathbf{n}(\%)]}\end{array}$ \\
\hline $\begin{array}{l}\text { Piperacillin - } \\
\text { Tazobactam }\end{array}$ & $52(50)$ \\
\hline Meropenem & $36(34.62)$ \\
\hline Metronidazole & $36(34.62)$ \\
\hline Linezolid & $23(22.12)$ \\
\hline Azithromycin & $21(20.19)$ \\
\hline Colistin & $17(16.35)$ \\
\hline Doxycycline & $17(16.35)$ \\
\hline $\begin{array}{l}\text { Amoxycillin - } \\
\text { Clavulanic acid }\end{array}$ & $15(14.42)$ \\
\hline Levofloxacin & $14(13.46)$ \\
\hline PolymyxinB & $14(13.46)$ \\
\hline Tigecycline & $14(13.46)$ \\
\hline
\end{tabular}

\section{DISCUSSION}

The patients in this study were in the age group of 20-80 years with the mean age of $51.95 \pm 16.83$ years. This may be because sepsis is common in the aging population due to multiple factors and declining immunity past middle age (Figure 1). ${ }^{13}$

Our data also showed that there were more Gram-negative $(50.96 \%)$ than Gram-positive isolates $(49.04 \%)$, an observation very similar to that stated earlier by Wattal et al. ${ }^{14}$ This is not surprising since the former are known to develop resistance more rapidly and extensively than the latter. ${ }^{15}$ Among the gram positive isolates, Coagulase negative staphylococci (CoNS) was the most common blood culture isolate (35.58\%) in this study (Figure 2). In an earlier study, Valles et al. have also reported CoNS as the commonest microorganism (20-30\%) causing blood stream infections (BSIs) among ICU patients. ${ }^{16}$ Thus it is obvious that CoNS is among one of the most common isolates from blood cultures in patients of ICU, though the extent of problem varies from region to region.

Interestingly, CoNS is also a very preventable cause of infection because CoNS isolates are often skin colonizers and appear in blood culture as common contaminants at the time of sample collection. ${ }^{17}$ In this study, both CoNS and Staphylococcus aureus showed resistance to penicillin in almost all patients, which is a similar to another study done by Sheth KV et al. ${ }^{13}$ In this study, S. aureus isolates were resistant to methicillin in 67\% patients (Table 1). Our findings are similar to earlier studies that report Methicillin Resistant Staphylococcus aureus (MRSA) in 47\% to 65\% of the isolates. These trends are indicative of the ever increasing resistance to beta lactams among CoNS. ${ }^{18}$

Klebsiella pneumoniae, Escherichia coli, Acinetobacter baumannii complex were the common gram negative isolates in this study (Figure 2). Even among Gram negative bacteria, resistance to Beta lactams or combination of Beta lactams with Beta lactamase inhibitors was a dominant feature (Table 2). The gram negative bacteria were however sensitive to Aminoglycosides, Colistin and Tigecycline to a variable extent, a trend very similar to that documented by Wattal et al. ${ }^{14}$

Authors found that Klebsiella pneumoniae was resistant to most of the commonly prescribed antibiotics like Piperacillin - Tazobactam, Meropenem, Amikacin and Tigecycline (Table 2). Authors also found that among the gram negative isolates, Klebsiella pneumoniae and Acinetobacter species have emerged as problematic species in this ICU. However, E. coli was sensitive to drugs like Minocycline, Ertapenem, Imipenem, Meropenem, Amikacin, Colistin and Tigecycline (Table 2). A similar trend, as observed in this study has also been reported by previous study. ${ }^{14}$

Therefore, currently, Colistin, Tigecycline and to some extent Aminoglycosides remains the only therapeutic options against gram negative isolates recovered from ICU patients.

The most frequently used antibiotics in this ICU were Piperacillin - Tazobactam (50\%) followed by Meropenem (34.62\%), Metronidazole (34.62 \%), Linezolid (22.12\%), Azithromycin (20.19\%), Colistin (16.35\%), Doxycycline (16.35\%), Amoxycillin - Clavulanic acid (14.42\%), Levofloxacin (13.46\%), Polymyxin B (13.46\%) and Tigecycline $(13.46 \%)$ (Table 3). A similar trend in usage pattern has been observed in another study. ${ }^{14}$

A lack of detailed data about resistance pattern in this region might have contributed to high use of resitant antibiotics in this setup. These results bring to light that a prior knowledge of the most common bacterial isolates and their antibiotic susceptibility patterns in a given region or a given ICU can help improve the prescribing patterns and support evidence based decisions to restrict or reduce the clinical availability of certain antibiotics. On studying the pattern of consumption of various antibiotics in this ICU, authors found that there is a predominant use of secondline antibiotics with broad spectrum of activity.

\section{CONCLUSION}

An establishment of antibiotic policy at national, state and hospital level are the need to promote prefer and judicious use of antibiotics and prevent emergence of drug resistance.

Funding: No funding sources Conflict of interest: None declared

Ethical approval: The study was approved by the Institutional Ethics Committee (SRHU/HIMS/ETHICS/2017/112) 


\section{REFERENCES}

1. Ibrahim NS, Ahmed F. Antimicrobial Activities of Some Synthetic Flavonoids. IOSR Journal of Applied Chemistry. 2014;7(5): -6.

2. Gangwar AS, Kumar N, Kothiyal P. Antibiotic prescription and cost patterns in an intensive care unit: a review of literature. The Pharma Innovation. 2012;1(7):68-72.

3. Rhodes A, Evans L, Alhazzani W, Levy M, Antonelli M, Ferrer R et al. Surviving sepsis campaign: international guidelines for management of sepsis and septic shock: 2016. Intensive Care Med. 2017;43(3):304-77.

4. Leekha S, Terrell CL, Edson RS. General Principles of Antimicrobial Therapy. Mayo Clin Proc. 2011;86(2):156-67.

5. Directorate General of Health Services policy. Ministry of Health and Family Welfare. National policy for containment of antimicrobial resistance India 2011. Available at: http://www.ncdc.gov.in/ab_policy.pdf/ Accessed August 2017.

6. World Health organization. Antimicrobial resistance. Available

at: http://www.who.int/mediacentre/factsheets/fs194/en/ Updated.April 2015/ Accessed September 2017.

7. Magiorakos PA, Srinivasan A, Carey RB, Carmeli Y, Falagas ME, Giske CG, et al. Multidrug-resistant, extensively drug-resistant and pandrug-resistant bacteria: an international expert proposal for interim standard definitions for acquired resistance. Clin Microbiol Infect. 2012;18:268-81.

8. Islam MA, Alam MM, Choudhury ME, Kobayashi N, Ahmed MU. Determination of Minimum Inhibitory Concentration (MIC) of Cloxacillin for selected isolates of Methicillin-Resistant Staphylococcus aureus (MRSA) with their antibiogram. Bangl J Vet Med. 2008;6(1):121-6.

9. Shrikala B, Kranthi K, Nafisa. A Prospective Study on Evaluation of Antibiotic Prescription Practices in an Intensive Care Unit of a Tertiary Care Hospital. J Clin Diagn Res. 2010;4(6):3387-91.

10. Balaji S, Karthik S, Pasupuleti M, Kannan G, Srinivasan D, Sekar U, et al. A Study on Evaluation of
Appropriateness of Antibiotics Used in the Intensive Care Unit Settings of a Tertiary Care Teaching Hospital. Int J Pharm Sci Rev Res. 2014;28(2):28-34.

11. Collee JG, Miles RS, Watt B. Tests for identification of bacteria. In collee JG, Fraser AG, Marmion BP, Simmons A, editors, Mackie and McCartney practical medical Microbiology. $14^{\text {th }}$ Ed. New York: Churchill Livingstone; 2007:131-149.

12. BioMerieux. Vitek 2- BioMerieux Clinical Diagnostics. Available at: http://www.biomerieuxdiagnostics.com/vitek-2/Accessed September 2017.

13. Sheth KV, Patel TK, Malek SS, Tripathi CB. Antibiotic sensitivity pattern of bacterial isolates from the intensive care unit of a tertiary care hospital in India. Trop J Pharm Res. 2012;11(6):991-9.

14. Wattal C, Raveendran R, Goel N, Oberoi JK, Rao BK. Ecology of blood stream infection and antibiotic resistance in intensive care unit at a tertiary care hospital in North India. Braz J Infect Dis. 2014;18(3):245-51.

15. Lepape A, Monnet DL. Experience of European intensive care physicians with infections due to antibiotic-resistant bacteria, 2009. European Communicable Disease Bulletin. 2008;14(45):429-33.

16. Valles J, Ferrer R. Bloodstream infection in the ICU. Infect Dis Clin North Am. 2009;23(3):557-69.

17. Weistein MP, Towns ML, Quartey SM, Mirrett S, Reimer LG, Parmigiani G, et al. The clinical significance of positive blood cultures in the 1990s: a prospective comprehensive evaluation of the microbiology, epidemiology, and outcome of bacteremia and fungemia in adults. Clin Infect Dis. 1997;24(4):584-602.

18. Ahmed SH, Daef EA, Badary MS, Mahmoud MA, Abd-Elsayed AA. Nosocomial blood stream infection in intensive care units at Assiut University Hospitals (Upper Egypt) with special reference to extended spectrum $\beta$-lactamase producing organisms. BMC Res Notes. 2009;2:76-86.

Cite this article as: Bhatia A, Kalra J, Kohli S, Kakati B, Kaushik R. Antibiotic resistance pattern in intensive care unit of a tertiary care teaching hospital. Int J Basic Clin Pharmacol 2018;7:906-11. 